\title{
Superconductivity and Single Crystal Growth of $\mathrm{Ni}_{0.05} \mathrm{TaS}_{2}$
}

X.D. Zhu ${ }^{a}$, Y.P. Sun ${ }^{\mathrm{a}, \mathrm{b}, *}$, S.B. Zhang ${ }^{\mathrm{a}}$, H.C. Lei ${ }^{\mathrm{a}}$, L.J. Li ${ }^{\mathrm{a}}$, X.B. Zhu ${ }^{\mathrm{a}}$, Z.R. Yang ${ }^{\mathrm{a}}$, W.H. Song ${ }^{\mathrm{a}}$, J.M.

$$
\text { Dai }^{\mathrm{a}}
$$

${ }^{a}$ Key Laboratory of Materials Physics, Institute of Solid State Physics, Chinese Academy of Sciences, Hefei 230031, People's Republic of China

${ }^{\mathrm{b}}$ High Magnetic Field Laboratory, Chinese Academy of Sciences, Hefei 230031, People's Republic of China

\begin{abstract}
Superconductivity was discovered in $\mathrm{Ni}_{0.05} \mathrm{TaS}_{2}$ single crystal. $\mathrm{Ni}_{0.05} \mathrm{TaS}_{2}$ single crystal was successfully grown via the $\mathrm{NaCl} / \mathrm{KCl}$ flux method. The obtained lattice constant $c$ of $\mathrm{Ni}_{0.05} \mathrm{TaS}_{2}$ is $1.1999 \mathrm{~nm}$, which is significantly smaller than that of $2 \mathrm{H}-\mathrm{TaS}_{2}(1.208 \mathrm{~nm})$. Electrical resistivity and magnetization measurements reveal that the superconductivity transition temperature of $\mathrm{Ni}_{0.05} \mathrm{TaS}_{2}$ is enhanced from $0.8 \mathrm{~K}\left(2 \mathrm{H}-\mathrm{TaS}_{2}\right)$ to $3.9 \mathrm{~K}$. The charge-density-wave transition of the matrix compound $2 \mathrm{H}-\mathrm{TaS}_{2}$ is suppressed in $\mathrm{Ni}_{0.05} \mathrm{TaS}_{2}$. The success of $\mathrm{Ni}_{0.05} \mathrm{TaS}_{2}$ single crystal growth via the $\mathrm{NaCl} / \mathrm{KCl}$ flux demonstrates that $\mathrm{NaCl} / \mathrm{KCl}$ flux method will be a feasible method for single crystal growth of the layered transition metal dichalcogenides.

PACS: 81.10.Dn; 74.70.-b

Keywords: A2. Single crystal growth; A2. Flux method; B1. Sulfides; B2. Superconducting materials
\end{abstract}

* Corresponding author. Tel.: +86 551559 2757; Fax: +86 5515591434.

E-mail address: ypsun@issp.ac.cn 


\section{Introduction}

Recently, several iron based supercondutors $\operatorname{LnOFePn}(L n=$ Rare earth, $P n=$ pnictide) $[1,2]$, $\mathrm{A}_{x} \mathrm{FeAs}$ with $\mathrm{ThCr}_{2} \mathrm{Si}_{2}$ structure $(\mathrm{A}$ is $\mathrm{A}=\mathrm{Ca}$, $\mathrm{Sr}$, or $\mathrm{Ba}$ with substitution of $\mathrm{A}$ by an alkali metal such as $\mathrm{K}$ or Cs.) [3-5] and $\alpha-\mathrm{FeSe}_{\mathrm{x}}$ [6] were discovered, which aroused a worldwide surge in searching for new superconductors. Interestingly, all of these compounds, as well as the copper-based high temperature superconductors [7,8], are layered structure materials. Layered transition metal dichalcogenides (TMDC), such as $\mathrm{TiSe}_{2}, \mathrm{NbSe}_{2}$ and $\mathrm{TaS}_{2}$, are well-known layered materials [9]. The structure of layered TMDC can be regarded as stacking of covalent coupled $\mathrm{X}-\mathrm{T}-\mathrm{X}$ ( $\mathrm{T}$, represents the transition metal, $\mathrm{X}=\mathrm{S}, \mathrm{Se}, \mathrm{Te}$ ) sandwiches, and the coupling between sandwiches is of weak van de Waals type. Organic molecules, alkali metals and transition metals can be intercalated into the van de Waals gap between the sandwiches [10-16]. 2H-TaS 2 undergoes a charge-density-wave (CDW) transition at $\sim 78 \mathrm{~K}$ and a superconducting transition at $\sim 0.8 \mathrm{~K}[17]$. The intercalation effects in $\mathrm{TaS}_{2}$ including superconductivity have attracted extensive efforts during the past decades $[10,14,16,18,19,20]$. As for 3d-transitional metal (3d-TM), superconductivity has been observed in Fe dilute alloys $\mathrm{Fe}_{0.05} \mathrm{TaS}_{2}$ [14] and copper intercalated $\mathrm{Cu}_{x} \mathrm{TaS}_{2}[21,22]$. Recently, superconductivity has also been discovered in $\mathrm{Cu}_{x} \mathrm{TiSe}_{2}$ [23, 24]. Whether superconductivity can be discovered in other $3 \mathrm{~d}-\mathrm{TM}_{x} \mathrm{TaS}_{2}$ becomes an intriguing question. The $\mathrm{Ni}$ intercalated compound $\mathrm{Ni}_{1 / 3} \mathrm{TaS}_{2}$ has been reported in some prior work $[25,26]$, and no superconductivity has been discovered in $\mathrm{Ni}_{\mathrm{x}} \mathrm{TaS}_{2}$ compounds. Thus, experiments were carried out to search for superconductivity in $\mathrm{Ni}_{\mathrm{x}} \mathrm{TaS}_{2}$ with $\mathrm{x}<0.1$.

Chemical-vapor-transport (CVT) is a traditional method for the single crystal growth of TMDC [27]. Flux method, which had rarely been applied in single crystal growth of TMDC, was introduced by M. Y. Tsay et al. in single crystal growth of $\mathrm{RuX}_{2}$ using Te as flux [28]. In this paper, we report the single crystal growth of $\mathrm{Ni}_{0.05} \mathrm{TaS}_{2}$ using the $\mathrm{NaCl} / \mathrm{KCl}$ flux method and its enhanced superconductivity.

\section{Experimental procedure}

First, $\mathrm{Ni}_{0.05} \mathrm{TaS}_{2}$ powder was synthesized by heating stoichiometric $\mathrm{Ni}(99.9 \%)$, Ta $(99.9 \%)$ and $\mathrm{S}(99.5 \%)$ powders in an evacuated quartz tube at $900^{\circ} \mathrm{C}$ for 4 days. The obtained $\mathrm{Ni}_{0.05} \mathrm{TaS}_{2}$ powder with 5 times $\mathrm{NaCl} / \mathrm{KCl}$ flux in weight were mixed and grounded thoroughly. Then, the 
mixed powders were sealed in an evacuated quartz tube. The tube was placed in a vertical furnace, heated to $850^{\circ} \mathrm{C}$ and maintained for 6 hours. Subsequently, the tube is cooled to $650^{\circ} \mathrm{C}$ in a rate of $2^{\circ} \mathrm{C} /$ hour and kept at $650^{\circ} \mathrm{C}$ for 2 days before the following furnace cooling. The grown single crystals were separated by resolving the $\mathrm{NaCl} / \mathrm{KCl}$ flux using deionized water. The dark blue, mirror-like plates in a typical size of $1.5 \times 1.5 \times 0.2 \mathrm{~mm}^{3}$ were obtained, and the photograph is shown in the inset of Fig. 1. The $2 \mathrm{H}-\mathrm{TaS}_{2}$ and $\mathrm{Cu}_{\mathrm{x}} \mathrm{TaS}_{2}$ single crystals mentioned in this paper were grown via the CVT method using iodine as an agent [22].

The composition of $\mathrm{Ni}_{0.05} \mathrm{TaS}_{2}$ single crystal was checked by energy dispersive $\mathrm{x}$-ray spectroscopy (EDS). Some single crystals were crushed into powder for the powder X-ray diffraction pattern (XRD) experiments. The single crystal XRD and powder XRD experiments were performed on a Philips X'pert PRO x-ray diffractometer with $\mathrm{Cu} \mathrm{K}_{\alpha}$ radiation at room temperature. $\mathrm{Ni}_{0.05} \mathrm{TaS}_{2}$. The electrical resistivity experiments were performed by the standard four-probe method using a quantum design physical property measurement system (PPMS) $(1.8 \mathrm{~K} \leq \mathrm{T} \leq 400 \mathrm{~K}, 0 \mathrm{~T} \leq$ $\mathrm{H} \leq 9 \mathrm{~T}$ ). Magnetization measurement as a function of temperature was performed in a quantum design superconducting quantum interference device (SQUID) system $(1.8 \mathrm{~K} \leq \mathrm{T} \leq 400 \mathrm{~K}, 0 \mathrm{~T} \leq \mathrm{H}$ $\leq 5 \mathrm{~T})$.

\section{Results and discussions}

The EDS pattern for the $\mathrm{Ni}_{0.05} \mathrm{TaS}_{2}$ single crystal is shown in Fig. 1. The determined mol ratio of $\mathrm{Ta}: \mathrm{S}$ is about $1: 2$. The nickel peak is evident in the EDS spectrum, and the determined mol ratio of the $\mathrm{Ni}$ : Ta is about $0.05: 1$, which is consistent with the nominal ratio of the raw materials.

Fig. 2 shows the single crystal XRD patterns for $\mathrm{Ni}_{0.05} \mathrm{TaS}_{2}$ and $2 \mathrm{H}-\mathrm{TaS}_{2}$ single crystals. The $2 \theta$ peak positions of $\mathrm{Ni}_{0.05} \mathrm{TaS}_{2}$ and $2 \mathrm{H}-\mathrm{TaS}_{2}$ are very close. This suggests that they have the same crystal structure and that their crystal surfaces are both parallel to $(00 l)$ crystal planes. The inset of Fig. 2 shows the magnification plots of the single crystal XRD patterns for $\mathrm{Ni}_{0.05} \mathrm{TaS}_{2}$ and $2 H-\mathrm{TaS}_{2}$. Obviously, compared with the $2 \theta$ peak positions of $2 \mathrm{H}-\mathrm{TaS}_{2}$, the $2 \theta$ peak positions of $\mathrm{Ni}_{0.05} \mathrm{TaS}_{2}$ shift to higher angle. The single XRD pattern for $\mathrm{Ni}_{0.05} \mathrm{TaS}_{2}$ reflects that the single crystal $\mathrm{Ni}_{0.05} \mathrm{TaS}_{2}$ is of good quality. Fig. 3 shows the powder XRD patterns for $\mathrm{Ni}_{0.05} \mathrm{TaS}_{2}$ and $2 \mathrm{H}-\mathrm{TaS}_{2}$. Apparently, the powder XRD patterns confirm that $\mathrm{Ni}_{0.05} \mathrm{TaS}_{2}$ and $2 \mathrm{H}-\mathrm{TaS}_{2}$ have the same structure. Meanwhile, the shift of the $2 \theta$ peak positions of $\mathrm{Ni}_{0.05} \mathrm{TaS}_{2}$ can be also observed in the inset of Fig. 3. According 
to the Bragg formula $2 d \sin \theta=\mathrm{n} \lambda$, the shift of the $(00 l)$ peak positions demonstrate the lattice constant $c$ is reduced in $\mathrm{Ni}_{0.05} \mathrm{TaS}_{2}$. In contrast, the copper intercalation in $\mathrm{Cu}_{\mathrm{x}} \mathrm{TiSe}_{2}$ [23] and $\mathrm{Cu}_{x} \mathrm{TaS}_{2}[21,22]$ result in the expansion of the lattice constant $c$ with increase of $x$ when $x<0.1$. The reduction of lattice constant $c$ suggests that $\mathrm{Ni}$ ions occupy the positions of $\mathrm{Ta}$ in $\mathrm{Ni}_{0.05} \mathrm{TaS}_{2}$.

The structural parameters of $\mathrm{Ni}_{0.05} \mathrm{TaS}_{2}$ were refined by the standard Rietveld technique [29]. Although the exact position of $\mathrm{Ni}$ ions can not be determined due to the low concentration and random distribution, better refinement can be obtained if $\mathrm{Ni}$ ions are assumed to occupy the position of Ta. This supports that $\mathrm{Ni}$ ions occupy the positions of $\mathrm{Ta}$ in $\mathrm{Ni}_{0.05} \mathrm{TaS}_{2}$. Fig. 4 shows the calculated XRD and the experimental XRD patterns. The structure parameters obtained from the best refinement are listed in Tab. 1. Due to the prefered orientation of $(00 l)$ crystal planes during the XRD experiment, the best $R_{p}$ value is relatively larger. The determined lattice constant $c$ of $\mathrm{Ni}_{0.05} \mathrm{TaS}_{2}$ is $1.1999(1) \mathrm{nm}$, while that of $2 \mathrm{H}-\mathrm{TaS}_{2}$ is $1.208 \mathrm{~nm}[22]$.

Fig. 5 shows the temperature dependence of electrical resistivity in $a b$ plane $\left(\rho_{a b}-T\right)$ for $\mathrm{Ni}_{0.05} \mathrm{TaS}_{2}$. The $\rho_{a b}-T$ curve shows a metallic behavior in the normal state and a nearly linear relation can be observed in the temperature range of $T>100 \mathrm{~K}$. The resistivity starts to drop at $T=3.9 \mathrm{~K}$, which indicates the superconductivity with $T_{\text {Conset }}=3.9 \mathrm{~K}$. The inset shows the enlarged view of the $\rho_{a b}-T$ curve near the superconducting region. The resistivity drops to zero at $T=2.2 \mathrm{~K}$ with a transition width $(10-90 \%)$ of about $1.4 \mathrm{~K}$. The wide superconducting transition has been also observed in $\mathrm{Fe}_{0.05} \mathrm{TaS}_{2}$ [14]. The large transition width may originate from the inhomogeniety.

Fig. 6 shows the temperature dependence of dc magnetic susceptibility for $\mathrm{Ni}_{0.05} \mathrm{TaS}_{2}$. The magnetic field of 10 Oe was applied parallel to the $c$ axis of a $\mathrm{Ni}_{0.05} \mathrm{TaS}_{2}$ plate. A sharp drop of magnetization at $2.2 \mathrm{~K}$ was observed for zero-field-cooling (ZFC) measurements, which further confirms the existence of superconductivity. The magnetic susceptibility does not saturate in the experimental temperature range.

Fig. 7 depicts the normalized resistivity $\left(\rho_{a b}(T) / \rho_{a b}(300 \mathrm{~K})\right.$ for $\mathrm{Ni}_{0.05} \mathrm{TaS}_{2}, \mathrm{Cu}_{0.08} \mathrm{TaS}_{2}$, $\mathrm{Cu}_{0.03} \mathrm{TaS}_{2}$ and $2 \mathrm{H}-\mathrm{TaS}_{2}$, respectively. The enlarged view of the low temperature region is shown in the inset of Fig. 7. Kinks can be observed in the resistivity curves for $\mathrm{Cu}_{0.03} \mathrm{TaS}$ and $2 \mathrm{H}-\mathrm{TaS}_{2}$, which reflects the CDW transitions [22]. While no sign of CDW transition can be observed for $\mathrm{Ni}_{0.05} \mathrm{TaS}_{2}$ and $\mathrm{Cu}_{0.08} \mathrm{TaS}_{2}$. The residual resistivity ratio (RRR), $\rho_{a b}(300 \mathrm{~K}) / \rho_{a b}(5 \mathrm{~K})$ for $\mathrm{Ni}_{0.05} \mathrm{TaS}_{2}$ is 1.6 , which is smaller than 3.1 for $\mathrm{Cu}_{0.08} \mathrm{TaS}_{2}$. The low $\mathrm{RRR}$ has been also observed in graphite intercalated 
compounds $\mathrm{CaC}_{6}(\mathrm{RRR} \approx 2)\left[30\right.$ ] and $\mathrm{Cu}_{x} \mathrm{TiSe}_{2}$ [23]. The scattering between the conducting carriers and the foreign atoms may be the origins of the observed low RRR in various intercalated compounds.

Combined with superconductors $\mathrm{Cu}_{\mathrm{x}} \mathrm{TiSe}_{2}$ [23], $\mathrm{Fe}_{0.05} \mathrm{TaS}_{2}$ [14] and $\mathrm{Cu}_{x} \mathrm{TaS}_{2}$ [21, 22], the superconductivity discovered in $\mathrm{Ni}_{0.05} \mathrm{TaS}_{2}$ implies that superconductivity may exist in other $3 \mathrm{~d}-\mathrm{TM}$ intercalated or dilute doped TMDC with low concentration. In addition, $\mathrm{NaCl} / \mathrm{KCl}$ flux method can be a feasible method for single crystal growth of the layered TMDC.

\section{Conclusion}

In summary, $\mathrm{Ni}_{0.05} \mathrm{TaS}_{2}$ single crystals were successfully grown via the $\mathrm{NaCl} / \mathrm{KCl}$ flux method. Structure analysis shows that the lattice constant $c$ of $\mathrm{Ni}_{0.05} \mathrm{TaS}_{2}$ is smaller than that of $2 \mathrm{H}-\mathrm{TaS}_{2}$, which may originate from the Ta replacement by Ni. Electrical resistivity and magnetization measurements reveal that the superconductivity transition temperature of $\mathrm{Ni}_{0.05} \mathrm{TaS}_{2}$ is enhanced from $0.8 \mathrm{~K}\left(2 \mathrm{H}-\mathrm{TaS}_{2}\right)$ to $3.9 \mathrm{~K}$. The $\mathrm{CDW}$ transition of the matrix compound $2 \mathrm{H}-\mathrm{TaS}_{2}$ is suppressed in $\mathrm{Ni}_{0.05} \mathrm{TaS}_{2}$. The observation of superconductivity in $\mathrm{Ni}_{0.05} \mathrm{TaS}_{2}$ implies that superconductivity may exist in other 3d TM intercalated or dilute doped TMDC with low concentration. In addition, the $\mathrm{NaCl} / \mathrm{KCl}$ flux method provides a potential method for single crystal growth of the layered transitional metal dichalcogenides.

\section{Acknowledgements}

This work is supported by the National Key Basic Research under contract No. 2006CB601005, 2007CB925002, and the National Nature Science Foundation of China under contract No.10774146, 10774147 and Director's Fund of Hefei Institutes of Physical Science, Chinese Academy of Sciences.

\section{References}

[1] Y. Kamihara, H. Hiramatsu, M. Hirano, R. Kawamura, H. Yanagi, T. Kamiya and H. Hosono, J. Am. Chem. Soc., 128 (2006) 10012.

[2] Y. Kamihara, T. Watanabe, M. Hirano and H. Hosono, J. Am. Chem. Soc. 130 (2008) 3296.

[3] M. Rotter, M. Tegel, D. Johrendt, I. Schellenberg, W. Hermes and R. Pöttgen, Phys. Rev. B 78 
(2008) 020503(R).

[4] M. Rotter, M. Tegel and D. Johrendt, Phys. Rev. Lett. 101 (2008) 107006.

[5] K. Sasmal, B. Lv, B. Lorenz, A. M. Guloy, F. Chen, Y.Y. Xue and C.W. Chu, Phys. Rev. Lett. $101(2008) 107007$.

[6] F.C. Hsu, J.Y. Luo, K.W. Yeh, T.K. Chen, T.W. Huang, Phillip M. Wu, Y.C. Lee, Y.L. Huang, Y.Y. Chu, D.C. Yan and M.K. Wu, Proc. Natl. Acad. Sci. U.S.A. 105 (2008) 14262.

[7] J.G. Bednorz and K.A. Müller, Z. Phys. B 64 (1986) 189.

[8] A.L. Patrick, N. Nagaosa and X.G. Wen, Rev. Mod. Phys. 78 (2006) 17.

[9] J.A. Wilson and A.D. Yoffe, Adv. Phys. 18 (1969) 193.

[10] R.H. Friend and A.D. Yoffe, Adv. Phys. 36 (1987) 1.

[11] F.R. Gamble, F.J. Di Salvo, R.A. Klemm and T.H. Geballe, Science 168 (1970) 568.

[12] D.W. Murphy, F.J. Di Salvo, G.W. Hull and J.V. Waszczak, Inorg. Chem. 15 (1976) 17.

[13] A. Schlicht, A. Lerf and W. Biberacher, Synthetic Metals 102 (1999) 1483.

[14] R.M. Fleming and R.V. Coleman, Phys. Rev. Lett. 34 (1975) 1502.

[15] S.F. Meyer, R.E. Howard, G.R. Stewart, J.V. Acrivos and T.H. Geballe, J. Chem. Phys. 62 (1975) 4411.

[16] A. Lerf, F. Sernetz, W. Biberacher and R. Schöllhorn, Mat. Res. Bull. 14 (1979) 797.

[17] J.M.E. Harper, T.H. Geballe and F.J. Di Salvo, Phys. Rev. B 15 (1977) 2943.

[18] F.J. Di Salvo, Jr, G.W. Hull, L.H. Schwartz, J.M. Voorhoeve and J.V. Waszczak, J. Chem. Phys. 59 (1973) 1922.

[19] A.D. Yoffe, Solid State Ionics 9\&10 (1983) 59.

[20] L. Fang, Y. Wang, P.Y. Zou, L. Tang, Z. Xu, H. Chen, C. Dong, L. Shan, and H.H. Wen, Phys. Rev. B 72 (2005) 014534.

[21] K.E. Wagner, E. Morosan, Y.S. Hor, J. Tao, Y. Zhu, T. Sanders, T.M. McQueen, H.W. Zandbergen, A.J. Williams, D.V. West and R.J. Cava, Phys. Rev. B 78 (2008) 104520.

[22] X.D. Zhu, Y.P. Sun, X.B. Zhua, X. Luo, B.S. Wanga, G. Li, Z.R. Yang, W.H. Song and J.M. Dai, J. Crystal Growth 311 (2008) 218.

[23] E. Morosan, H.W. Zandbergen, B.S. Dennis, J.W. G. Bos, Y. Onose, T. Klimczuk, A.P. Ramirez, N.P. Ong and R.J. Cava, Nature Physics 2 (2006) 544.

[24] G. Wu, H.X. Yang, L. Zhao, X.G. Luo, T. Wu, G.Y. Wang and X.H. Chen, Phys. Rev. B 76 
(2007) 024513.

[25] J.M. van den Berg and P. Cossee, Inorg. Chim. Acta 2 (1968) 143.

[26] B. van Laar, H.M. Rietveld and D.J.W. Ijdo, J. Solid State Chem. 3 (1971) 154.

[27] D.E. Moncton, J.D. Axe and F.J. DiSalvo, Phys. Rev. B 16 (1977) 801.

[28] M.Y. Tsay, J.K. Huang, C.S. Chen and Y.S. Huang, Mater. Res. Bull. 30 (1995) 85.

[29] D. B. Wiles and R. A. Young, J. Appl. Crystallogr. 14 (1981) 149.

[30] E. Jobiliong, H.D. Zhou, J.A. Janik, Y.J. Jo, L. Balicas, J.S. Brooks and C.R. Wiebe, Phy.

Rev. B 76 (2007) 052511. 
Table captions:

Table 1. The refined structure parameters of $\mathrm{Ni}_{0.05} \mathrm{TaS}_{2}$ : Space group (S.G.), lattice constant $a$, lattice constant $c, R_{p}$ value, $R_{w p}$ value, and the atom positions.

Tables:

\begin{tabular}{cc}
\hline Structure parameters of $\mathrm{Ni}_{0.05} \mathrm{TaS}_{2}$ \\
\hline S.G. & $P 6_{3} / m m c$ \\
$c(\mathrm{~nm})$ & $0.33128(2)$ \\
$c(\mathrm{~nm})$ & $1.1999(1)$ \\
$R_{p}$ & 11.1555 \\
$R_{w p}$ & $(0,0,1 / 4)$ \\
$\mathrm{Ta} / \mathrm{Ni}$ & $(1 / 3,2 / 3,0.1206)$ \\
$\mathrm{S}$ &
\end{tabular}

Table 1 X. D. Zhu et al. 
Figure Captions:

Fig. 1. The EDS pattern for $\mathrm{Ni}_{0.05} \mathrm{TaS}_{2}$. Inset: photograph of the grown $\mathrm{Ni}_{0.05} \mathrm{TaS}_{2}$ single crystals.

Fig. 2. The single crystal XRD patterns for $\mathrm{Ni}_{0.05} \mathrm{TaS}_{2}$ and $2 H-\mathrm{TaS}_{2}$. The inset shows the magnification plot of (006) diffraction peak.

Fig. 3. The powder XRD patterns for $\mathrm{Ni}_{0.05} \mathrm{TaS}_{2}$ and $2 \mathrm{H}-\mathrm{TaS}_{2}$. The inset shows the magnification plot of (006) diffraction peak.

Fig. 4. The experimental and calculated XRD patterns for $\mathrm{Ni}_{0.05} \mathrm{TaS}_{2}$. Cross: the experimental pattern; upper solid line: the calculated XRD pattern; bar: the calculated peak positions; the lowest line: the difference of the experimental and calculated XRD patterns.

Fig. 5. The temperature dependence of the in-plane resistivity $\left(\rho_{a b}-T\right)$ for $\mathrm{Ni}_{0.05} \mathrm{TaS}_{2}$. The inset shows the $\rho_{a b}-T$ curve near the superconducting region. The arrow shows the zero resistance transition temperature.

Fig. 6. The temperature dependence of ZFC susceptibility measured in an applied field of $10 \mathrm{Oe}$ parallel to $c$ axis for $\mathrm{Ni}_{0.05} \mathrm{TaS}_{2}$. The arrow shows the transition temperature $T_{\mathrm{C}}$.

Fig. 7. The temperature dependence of the normalized resistivity $\left(\rho_{a b}(T) / \rho_{a b}(300 \mathrm{~K})\right)$ for $\mathrm{Ni}_{0.05} \mathrm{TaS}_{2}$, $\mathrm{Cu}_{0.08} \mathrm{TaS}_{2}, \mathrm{Cu}_{0.03} \mathrm{TaS}_{2}$ and $2 \mathrm{H}-\mathrm{TaS}_{2}$. The inset shows the enlarged view of the low temperature. 